\title{
Combination of p53 expression and p21 loss has an independent prognostic impact on sporadic colorectal cancer
}

\author{
AURELIA NOSKE $^{1}$, SYBILLE LIPKA $^{2}$, JAN BUDCZIES ${ }^{1}$, KATHRIN MÜLLER ${ }^{2}$, \\ CHRISTOPH LODDENKEMPER ${ }^{3}$, HEINZ JOHANNES BUHR ${ }^{2}$ and MARTIN KRUSCHEWSKI ${ }^{2}$ \\ ${ }^{1}$ Institute of Pathology, Charité University Hospital Berlin, Campus Mitte; ${ }^{2}$ Department of Surgery and \\ ${ }^{3}$ Institute of Pathology, Charité University Hospital Berlin, Campus Benjamin Franklin, Berlin, Germany
}

Received February 11, 2009; Accepted March 30, 2009

DOI: $10.3892 /$ or_00000398

\begin{abstract}
There is no clear evidence on the prognostic and predictive value of abnormal p53 expression in colorectal cancer. The major downstream protein, p21, a cell cycle inhibitor, is transcriptionally regulated by $\mathrm{p} 53$. The prognostic impact of $\mathrm{p} 21$ expression in colorectal carcinomas is still under debate. In this study, we investigated the expression of p21 and p53 in a prospective cohort of 116 sporadic colorectal carcinomas at UICCII/III stage. We observed an expression of p21 in $26 \%$ and p53 in $63 \%$ of the carcinomas by immunohistochemistry. Patients with p21negative colorectal carcinomas had a significant better recurrence-free and overall survival than patients with p21positive carcinomas $(\mathrm{p}=0.02$ and $\mathrm{p}=0.005)$. Expression of $\mathrm{p} 53$ was related to a better overall survival $(\mathrm{p}=0.048)$. The combination of $\mathrm{p} 21$-negative/p53-positive expression was significantly related to better recurrence-free and overall survival ( $\mathrm{p}=0.007$ and $\mathrm{p}=0.0001)$ and gained independent prognostic significance (HR: $3.4, \mathrm{p}=0.01$ ). Moreover, patients with combined $\mathrm{p} 21 \% / \mathrm{p} 53^{+}$expression had a remarkable benefit in overall survival after adjuvant chemotherapy as compared to the $\mathrm{p} 21^{\%} / \mathrm{p} 53^{-}$subgroup (HR: 3.6, $\mathrm{p}=0.027$ ). Our data suggest that the assessment of both p53 and p21 expression may provide prognostic information in colorectal cancer patients. This combination might be helpful to identify patients who could benefit from chemotherapy.
\end{abstract}

\section{Introduction}

Colorectal cancer is the third most common cancer in both genders in the Western world with an estimated number of 112,340 new cases and 52,180 estimated deaths in the USA in 2007 (1). The five-year survival rates reported for patients

Correspondence to: Dr Martin Kruschewski, Department of Surgery, University Hospital Charité Campus Benjamin Franklin, Hindenburgdamm 30, 12200 Berlin, Germany

E-mail: martin.kruschewski@charite.de

Key words: p21, p53, colorectal cancer, prognosis, adjuvant therapy with stage II and III colorectal cancer vary between 92 and $67 \%$ (SEER Cancer Statistics). The pathologic tumor stage represents the most important prognostic factor and generally survival decreases with later stage cancers. The association of stage IIIa colon cancer with statistically significantly better survival than stage IIb (83 vs. $72 \%$ ) in the AJCC sixth edition system may reflect current clinical practice, in which stage III patients receive chemotherapy but stage II patients generally do not (2). Patients with UICC stage III carcinomas will benefit from adjuvant chemotherapy in only $5-15 \%$ and the majority of patients who undergo adjuvant therapy will be overtreated $(3,4)$. Colorectal cancer is considered relatively resistent to chemotherapy (5). Various chemotherapeutic approaches such as treatment with 5-fluorouracil (5-FU) have led only to a weak to moderate decline in mortality. Therefore, additional parameters such as molecular biomarkers are needed to identify patients who will really benefit from adjuvant anti-cancer treatment.

Alterations in different molecular signalling pathways are involved in initiation and progression of colon cancer, such as e.g. p53 ('suppressor-pathway'), mismatch repair genes ('mutator-pathway') and EGFR-KRAS-ERK-pathway. The activation status of certain molecules can influence patient prognosis. Thus, it has been shown, that patients with mismatch repair gene mutations or microsatellite instable (MSI high) colorectal carcinomas have a better overall survival than those without these genetic defects (6). Moreover, patients with MSI high colorectal carcinomas had no benefit from 5-FU-based adjuvant chemotherapy, whereas patients with MSI low/MSS tumors did benefit from this therapy (7). Currently, targeted therapy with an anti-EGFR-antibody is applied in metastasized colorectal cancer. Patients will have a benefit of anti-EGFR therapy if a mutation in the oncogene KRAS can be excluded (8).

Although the p53 expression is abnormal in $>50 \%$ of the colorectal carcinomas, the prognostic role of the most intensively studied p53 tumor suppressor gene is contradictory. Patients whose tumors are associated with abnormal p53 protein have both reduced and improved outcome. The value in predicting reponse to chemotherapy is not well known. The p53 protein induces temporary cell cycle arrest, inhibits cell growth and contributes to apoptosis by regulation of $\mathrm{p} 21$. The p21/WAF1 cyclin-dependent kinase inhibitor blocks the transition from G1 to S-phase and inhibits cell proliferation. 
Thus, $\mathrm{p} 21$ protein serves as a surrogate marker for $\mathrm{p} 53$ protein alterations. While there are several controversial studies on p53, the expression of p21 and its significance for the prognosis in colorectal cancer as well as predictive function on the outcome of adjuvant therapy is not well elucidated.

We carried out this study to investigate the expression of p53 and p21 in a prospective cohort of patients with colorectal carcinomas stage II/III and to evaluate the prognostic significance as well as the influence on the outcome of adjuvant 5-FU-based chemotherapy in colon cancer and radiochemotherapy in rectal cancer.

\section{Patients and methods}

Study population and tissue samples. Immunohistochemical examination of p53 and p21 was performed retrospectively on tissue samples taken for routine diagnostic and therapeutic purposes. Curatively resected colorectal carcinomas of 116 patients who were diagnosed at the Institute of Pathology, Charité Hospital Berlin (Campus Benjamin Franklin) between 1995 and 2001 were included in the study. Only patients with primary colorectal adenocarcinomas and no other known malignancies and no preoperative radiochemotherapy were included. The tissue specimens consisted of 37 colon carcinomas and 79 rectum carcinomas as well as adjacent normal mucosa. Tissue samples were fixed in $4 \%$ neutralbuffered formaldehyde, embedded in paraffin and histopathological evaluation was performed on standard hematoxylin and eosin sections. The stage of tumors was assessed according to the UICC staging system. Tumor differentiation was determined according to the WHO guidelines. For all patients a minimum of 12 lymph nodes were investigated. Clinical follow-up data were available for all patients. The mean follow-up time of patients was 52 months.

Data on postoperative chemotherapy are known from 114 patients and on radiotherapy in 113 cases. Patients were treated according to the guidelines of the German Cancer Society. Thus, patients with colon cancer UICC stage III were administered chemotherapy (5-FU/folic acid). Postoperative radiochemotherapy (5-FU/folic acid and radiation doses of $45 \mathrm{~Gy}$ ) was administered to patients with rectal carcinomas in UICC stage II and III.

Immunohistochemistry. Immunohistochemistry was performed according to standard procedures on parraffin sections containing both normal mucosa and the invasive tumor front. Briefly, slides were boiled in citrate buffer ( $\mathrm{pH} \mathrm{6.0)} \mathrm{in} \mathrm{a}$ pressure cooker for $5 \mathrm{~min}$ and incubated with a monoclonal anti-p21 ${ }^{\mathrm{WAF} 1 / \mathrm{Cip} 1}$ antibody (Clone SX118, Dako) at 1:50 for $1.5 \mathrm{~h}$ at room temperature as well as a monoclonal anti-p53 antibody (Clone DO-7, Dako) at 1:100 for $1 \mathrm{~h}$ at room temperature, followed by incubation with a biotinylated anti-mouse secondary antibody and the multilink biotinstreptavidin-amplified detection system (Biogenex, San Ramon, CA, USA). Staining was visualized using a fast-red chromogen system (Immunotech, Hamburg, Germany). Appropriate positive and negative controls were included in each run of immunostaining. The p21 and p53 immunostaining in tumor cells was evaluated independently by two authors (A.N. and S.L.), who were blinded to patient outcome.
Discordant cases were discussed on a multihead microscope until a final decision was reached. Expression of p21 and p53 was evaluated according to the percentage of positive tumor cell nuclei. The immunoreactivity of p21 was categorised in negative expression $(<5 \%$ of the tumor cells) and positive expression ( $>5 \%$ of the tumor cells are positive). The percentage of p53-positive cells was scored as: $1(0-10 \%)$; $2(11-25 \%) ; 3(26-50 \%)$; and 4 (>50\%). For further analyses, a cutoff point of $>25 \%$ positive cells was defined. All immunoreactive nuclei were regarded as positive irrespective of the staining intensity. Negative controls were performed by omitting the primary antibody.

Statistical analysis. The statistical significance of the association between p21 and p53 status as well as clinicopathological parameters was assessed by Fisher's exact test. The probability of overall survival as a function of time was determined by the Kaplan-Meier method. Differences in survival curves were compared by the log-rank test. Cox proportional hazard models were fitted in order to calculate hazard ratios and to carry out multivariate survival analyses. Generally, p-values $<0.05$ were considered significant, hazard ratios are reported with $95 \%$ confidence intervals. For the statistical evaluation the SPSS software Version 13.0 was used.

\section{Results}

Clinical and pathological characteristics of colorectal carcinomas. Colorectal carcinoma specimens from 116 patients were investigated for immunoreactivity of p21 and p53. The mean age of patients at surgery was 64 years (range 38-89). Forty-eight patients were female and 68 male. The majority of tumors were diagnosed in tumor stage pT3 (89 cases, 76.6\%). Most carcinomas (52.6\%) were poorly differentiated. In total, 36 patients $(31 \%)$ had no lymph node metastasis (pN0), whereas 80 patients $(69 \%)$ had metastases in lymph nodes. Clinico-pathological features are summarized in Table I. Multivariate analysis revealed nodal- and metastasis status as the best discriminating factors regarding prognosis in our study cohort (Table II).

Expression of p21 and p53 in colorectal cancer. Immunohistochemical analysis of p21 was performed on 116 colorectal carcinomas (Fig. 1A). Positive expression of p21 was defined as a nuclear staining reaction in $>5 \%$ of the tumor cells according to other publications (9). We observed no p21 expression in 8 carcinomas, a nuclear expression in $<5 \%$ of the tumor cells in 77 carcinomas and a nuclear expression in $>5 \%$ in 31 colorectal carcinomas. In adjacent colorectal mucosa a p21 expression was found only in the epithelium of the upper part and surface. For p53, expression analysis was performed on 114 colorectal carcinomas (Fig. 1B). Positive expression of p53 was defined as a nuclear staining of $>25 \%$ tumor cells. According to the scoring sytem, we found a weak expression (0-10\% of the cells) in 30 tumors, a moderate expression (11-25\% of the cells) in 12 cases and a strong nuclear immunoreaction ( $>25 \%$ of the cells) in 72 colorectal carcinomas. In adjacent normal mucosa no p53 expression was observed. 
Table I. Clinicopathological characteristics of 116 patients with colorectal cancer.

\begin{tabular}{lr}
\hline Characteristic & All cases n (\%) \\
\hline pT & \\
pT1 & $2(1.7)$ \\
pT2 & $6(5.2)$ \\
pT3 & $89(76.7)$ \\
pT4 & $19(16.4)$ \\
pN & \\
pN0 & $36(31)$ \\
pN1 & $44(37.9)$ \\
pN2 & $27(23.3)$ \\
pN3 & $9(7.8)$ \\
Histological grade & \\
G1 & $1(0.9)$ \\
G2 & $54(46.6)$ \\
G3 & $61(52.6)$ \\
UICC stage & \\
II & $36(31)$ \\
III & $80(69)$ \\
Location & \\
Colon & \\
Rectum & $37(31.9)$ \\
\hline & $79(68.1)$ \\
\hline
\end{tabular}

Table II. Cox regression analysis under inclusion of clinicopathological factors $(n=114)$.

\begin{tabular}{|c|c|c|}
\hline \multirow[b]{2}{*}{ Parameter } & \multicolumn{2}{|c|}{ Overall survival } \\
\hline & $\mathrm{HR}(95 \% \mathrm{CI})$ & P-value \\
\hline \multicolumn{3}{|l|}{ Tumor stage } \\
\hline $\mathrm{pT} 1 / \mathrm{pT} 2$ & 1.000 & \\
\hline $\mathrm{pT} 3 / \mathrm{pT} 4$ & $1.63(0.49-5.37)$ & 0.425 \\
\hline \multicolumn{3}{|l|}{ Lymph node status } \\
\hline $\mathrm{pN} 0 / \mathrm{pN} 1$ & 1.000 & \\
\hline $\mathrm{pN} 2 / \mathrm{pN} 3$ & $2.71(1.45-5.08)$ & 0.002 \\
\hline \multicolumn{3}{|l|}{ Histologic grade } \\
\hline $\mathrm{G} 1 / \mathrm{G} 2$ & 1.000 & \\
\hline G3 & $1.11(0.59-2.11)$ & 0.746 \\
\hline \multicolumn{3}{|l|}{ Metastasis status } \\
\hline M0 & 1.000 & \\
\hline M1 & $3.37(1.87-6.08)$ & $<0.001$ \\
\hline \multicolumn{3}{|l|}{ Age at diagnosis } \\
\hline \multicolumn{3}{|l|}{ Expression } \\
\hline $\mathrm{p} 21^{-/} \mathrm{p} 53^{+}$ & 1.000 & \\
\hline $\mathrm{p} 21^{+} / \mathrm{p} 53^{-}, \mathrm{p} 21^{+} / \mathrm{p} 53^{+}, \mathrm{p} 21^{-/} / \mathrm{p} 53^{-}$ & $3.4(1.63-7.24)$ & 0.001 \\
\hline
\end{tabular}

$\mathrm{HR}$, risk ratio and $95 \% \mathrm{CI}, 95 \%$ confidence interval.
Relationship between $p 21, p 53$ and clinicopathological features. There was no relation between p21 and p53 expression $(\mathrm{p}=0.19)$. Colorectal carcinomas of patients without p21 expression were significantly associated with rectal tumor localization compared to tumors localized in colon $(\mathrm{p}=0.003)$. About half of the colon carcinomas (46\%) were p21-positive compared to only $18 \%$ of the rectum carcinomas. There was no significant relationship to any other clinicopathological parameter such as gender, age, tumor stage, tumor differentiation and nodal status. Concerning p53 expression, we did not find any significant association with clinicopathological parameters.

Relationship between p21, p53 and survival. In univariate Kaplan-Meier analysis, patients with p21-negative carcinomas had a significantly better recurrence-free $(\mathrm{p}=0.02)$ and overall survival $(\mathrm{p}=0.005)$ than patients with $\mathrm{p} 21$-positive carcinomas (Fig. 2A and B). Patients with carcinomas, localized in colon and negative p21 expression had a better recurrence-free $(p=0.046)$ and overall survival $(p=0.002)$ as compared to patients with positive p21 expression. In univariate analysis, patients with p53-positive carcinomas had a significant better overall survival than patients without p53 expression $(p=0.048)$. No differences were found concerning the progression-free survival $(\mathrm{p}=0.161)$. Patients with p53-positive rectal carcinomas had a better overall survival as compared to patients without p53 expression $(\mathrm{p}=0.002)$.

Relationship between $p 21, p 53$, adjuvant therapy and patient prognosis. Patients with p21-negative colorectal carcinomas and adjuvant therapy had a significantly better overall survival than patients without therapy $(p=0.017)$. Patients without therapy were exposed to a significantly higher risk, $\mathrm{HR}=2.7$ (CI 1.2-6.4). The hazard ratio adjusted for patient age, tumor stage, lymph node and metastasis status and histological grade was $\mathrm{HR}_{\mathrm{adj}}=1.83(\mathrm{CI} 0.63-5.4, \mathrm{p}=0.271)$. There was no significant impact on the recurrence-free survival $(p=0.239)$. Concerning the $p 53$ expression, no differences in recurrence-free and overall survival were observed in patients who received adjuvant therapy $(\mathrm{p}>0.05)$.

Association of combined p21/p53 expression and prognosis. Since the regulation of p21 is partly mediated by p53, we analyzed the combination of p21 and p53 expression with patient survival. Thus, we found a significantly better recurrence-free $(\mathrm{p}=0.007)$ and overall survival $(\mathrm{p}<0.0001)$ in p21-negative/ p53-positive carcinomas as compared to other

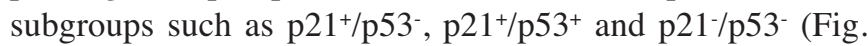
$3 \mathrm{~A}$ and $\mathrm{B})$. Cox analysis resulted in a higher risk for the latter patient group with $\mathrm{HR}=3.6$ (CI 1.8-7.3). When adjusted for patient age, tumor stage, lymph node and metastasis status and histological grade the hazard ratio remained significantly greater than one, $\mathrm{HR}_{\mathrm{adj}}=3.4$ (CI 1.6-7.2, p=0.001).

In this study, we have shown a significantly better overall survival after adjuvant therapy in patients with p21-negative carcinomas. Therefore, we investigated the role of the p53 expression status on the effects of postoperative therapy. We observed a better overall survival in patients with $\mathrm{p} 21^{-/} / \mathrm{p} 53^{+}$ carcinomas as compared to $\mathrm{p} 21^{-} / \mathrm{p} 53^{-}$carcinomas (log-rank, 

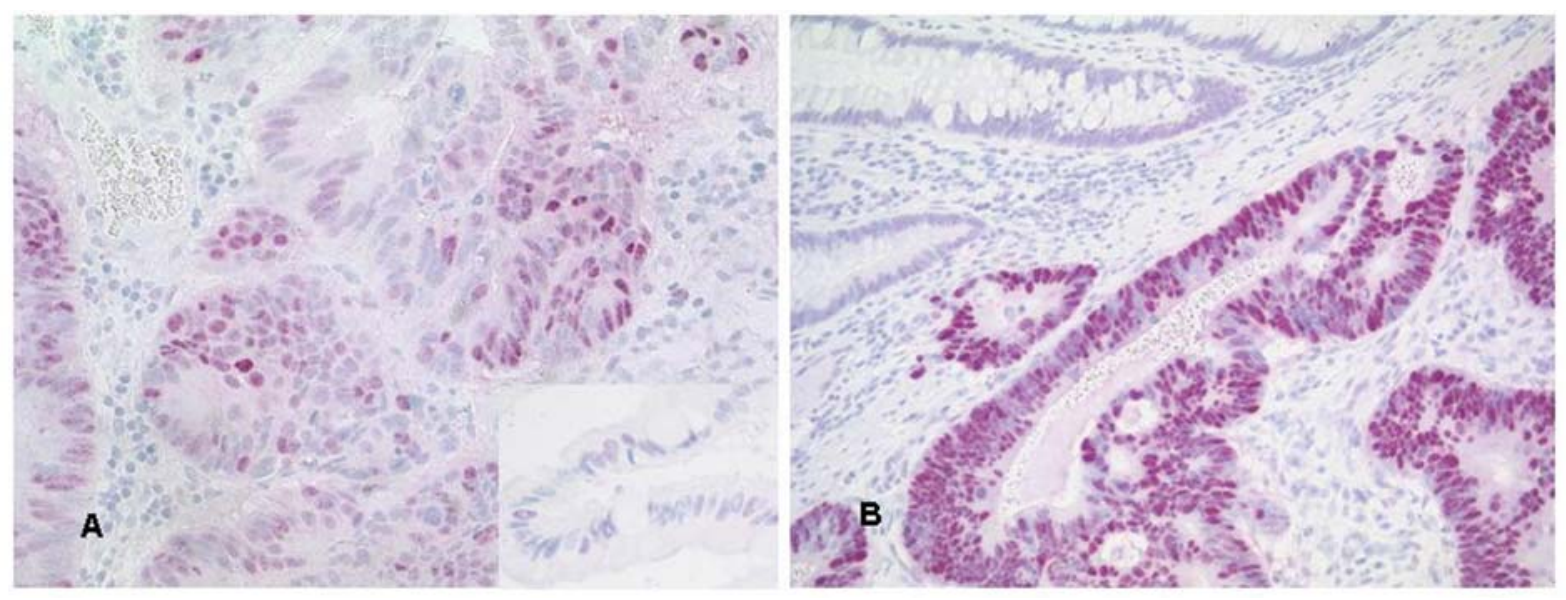

Figure 1. Expression of p21 and p53 in colorectal cancer. Nuclear expression of p21 in a colorectal adenocarcinoma. The insert shows superficial epithelial cells from adjacent normal mucosa with a weak nuclear staining (A). Strong nuclear expression of p53 in $>50 \%$ of an adenocarcinoma of the colon (right side) and adjacent normal colon mucosa without p53 expression on the left side (B).
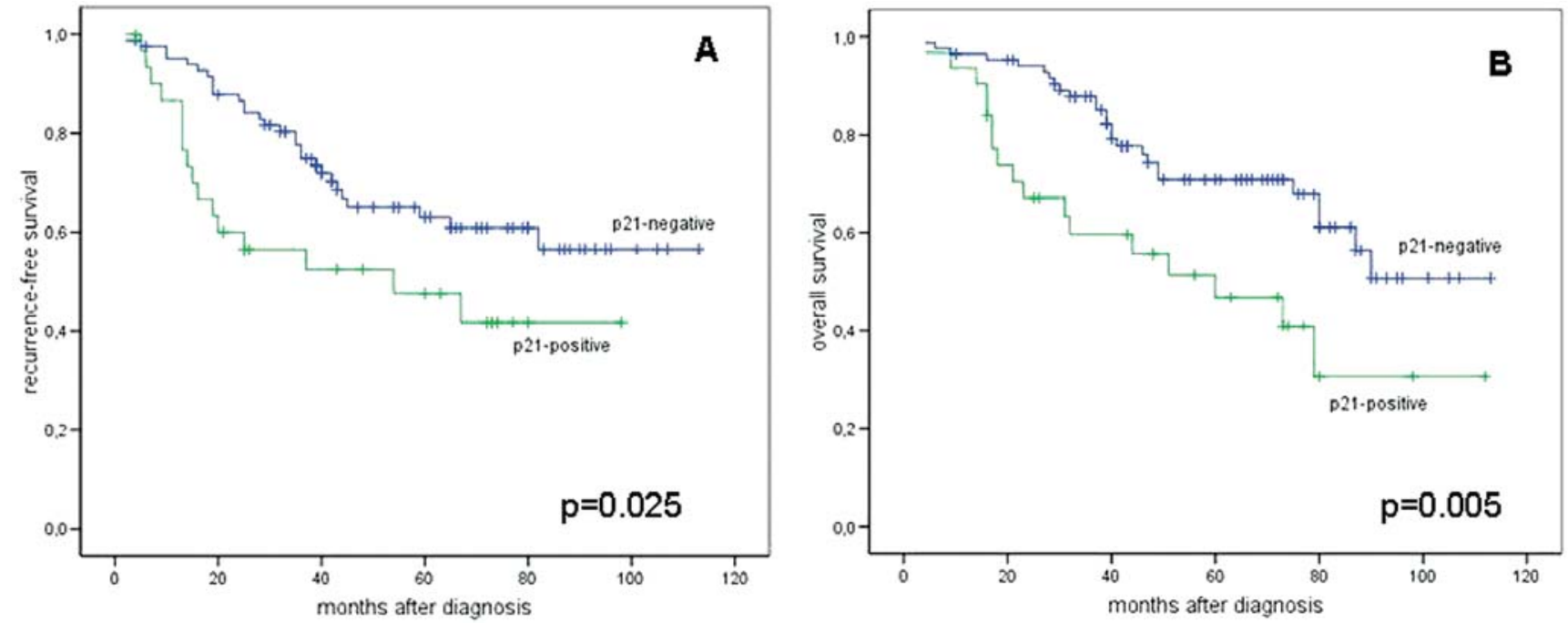

Figure 2. Univariate Kaplan-Meier survival analyses. Expression of p21 is related to better recurrence-free survival (A) and overall survival (B).
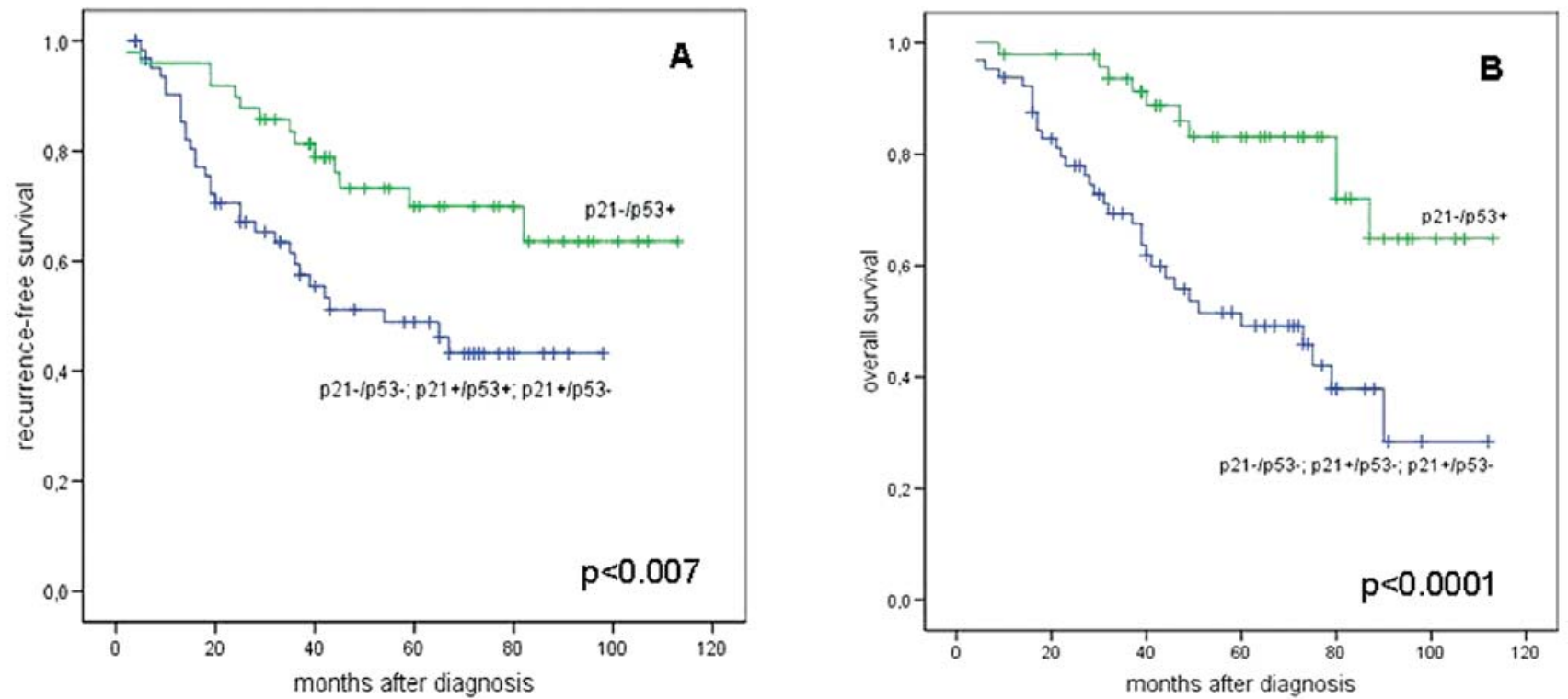

Figure 3. Univariate Kaplan-Meier survival analyses. The combination of p21\%/p53+ expression is associated with better recurrence-free (A) and overall survival (B). 
$\mathrm{p}=0.012)$. The $\mathrm{p} 53$-negative patients were exposed to higher risk, $\mathrm{HR}=3.5$ (CI 1.2-9.8) and $\mathrm{HR}_{\mathrm{adj}}=3.6$ (CI 1.2-11.6) when corrected for patient age, tumor stage, lymph node and metastasis status and histological grade.

\section{Discussion}

In this study, we hypothesized that alterations in p53 and p21 expression may provide prognostic information on colorectal cancer patients, particularly with regard to the adjuvant therapy.

p53 expression and relation to clinicopathological factors and patient outcome. We observed an expression of p53 in $63 \%$ of the colorectal carcinomas which is in line with previous studies, where a range of 40 to $81 \%$ p53 positivity was found (10-13). This wide range depends on different techniques used in these studies, such as different antibodies, scoring systems, cutoff-values and study populations. Inactive and mutated p53 protein accumulates in the nucleus and can be detected by immunohistochemistry. Antibodies used for immunohistochemistry can detect both the wild-type and the mutated p53 protein. Since, the wild-type occurs only at low detection levels and has a short half-life, it is widely accepted that most commercial antibodies are suitable for detection of abnormal p53. Since, this method has been shown to have both false positives and false negatives for the assessment of TP53 mutations (14), a higher threshold of p53 positivity was suggested to use for prediction of TP53 gene mutations (15).

We did not find any relationship to clinicopathological features as other authors did (12,16-18). In contrast, some studies reported a close correlation between p53 overexpression and poor prognostic markers such as lymph node metastasis and invasion depth (19-21). Whereas others found a relation to better differentiated tumors and location in the distal colon and rectum (22).

In our study, patients with p53 expression had a better overall survival $(\mathrm{p}=0.048)$ which is in line with previous studies $(9,22,23)$. These studies partly include large patient cohorts and long follow-up periods. A trend for improved overall survival for patients with p53 mutations in distal colonic carcinomas analyzed by single-strand conformation polymorphism analysis (SSCP) was also shown (23). Other studies reported an unfavourable prognosis in patients with p53-positive carcinomas $(12,13,24-28)$, but only few multivariate analyses yielded a significant result in these studies (25). However, other authors did not found a correlation of p53 and prognosis $(11,16,29,30)$. Additionally, we observed that patients with $\mathrm{p} 53$-positive carcinomas tended to have a better recurrence-free survival. In contrast, other studies found an association of p53 positivity and higher risk of tumor relapse as well as no relation to tumor recurrence $(12,13,27,29,31)$. The discrepancies between patient outcomes may depend on the tumor location (proximal vs. distal), p53 mutation site, tumor type and response to adjuvant therapy.

p21 expression and association with clinicopathological factors and patient outcome. In the present study, we observed a positive p21 expression in $26 \%$ of the colorectal carcinomas. Similar results were found by Fu et al (32) in rectal carcinomas. While other studies reported on p21 expression in 36 to $68 \%$ $(27,30,33,34)$. The different expression levels may depend on the different methods used, e.g. variable scoring systems. Further, we found a significant association of p21-negative carcinomas and rectal localization. Ricciardiello et al (10) reported an enhanced p21 expression in the right colon. Apart from these studies, data on p21 and its relationship to clinical and pathological findings are rare. Here, we observed a significantly better overall- and recurrence-free survival in patients with negative p21 colorectal carcinomas. In contrast, a negative p21 status was associated with higher risk of tumor relapse, poorer clinical course and lymph node and liver metastases in colorectal cancer $(27,35,36)$. Positive p21 expression correlated significantly with better recurrence-free survival and was shown to be an independent prognostic factor in colorectal cancer $(27,33)$. In rectal carcinomas, no association between p21 expression and clinicopathological features or survival was found (37).

Data on the impact of p21 expression on patient outcome in different cancer types is variable. Thus, expression of p21 has been correlated directly or inversely or not at all in survival of patients with invasive breast carcinomas (Cornfield et al) (38). The wide variety of stimuli that effect p21 expression through both p53-mediated and non-p53 pathways may account for the disparate observations.

Impact of p53 and p21 expression as well as adjuvant therapy on prognosis. In our study, patients with p53-positive carcinomas had a better (not significant) survival independent of adjuvant chemotherapy than p53-negative cases. Patients with left-sided carcinomas, Dukes C stage and normal p53 appear to benefit from adjuvant therapy rather than those with p53 mutation (23). Patients with stage III colon cancer and no p53 expression benefit from adjuvant 5-FU/ levamisole, whereas those with p53 overexpression do not (39). Previous studies have shown that the presence of p53 mutation failed to predict which patients would benefit from 5-FU-based adjuvant therapy (40). Abnormal p53 appears to be of no value in predicting response to chemotherapy alone, since the effects of p53 on the response to cytotoxic drugs, radiation and chemoradiation are complex (40). No significant association between p53 status and adjuvant chemotherapy was found in several colorectal cancer studies $(16,41)$. In this study, we demonstrated to our knowledge for the first time a significant better overall survival in patients with p21-negative carcinomas after adjuvant therapy. Similarly, negative p21 expression was associated with better recurrence-free survival after neoadjuvant treated in rectal carcinomas (42).

Relation of p53 and p21. In this study, we did not observe an association between p53 and p21 expression which is in line with other studies (43). In contrast, colorectal carcinomas with loss of p21 (50\% of 737 cases) exhibited a significantly higher frequency of p53 expression in a large prospective study. Both p21 negativity and p53 positivity were inversely associated with high microsatellite instability (MSI-H), CpG island methylator phenotype (CIMP-high) and BRAF mutations (15). Other authors reported an inverse correlation between these proteins $(10,27)$.

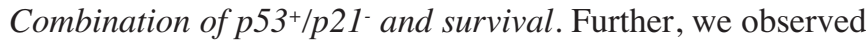
a better progression-free and overall survival in patients with 
p21-negative/p53-positive colorectal carcinomas as compared

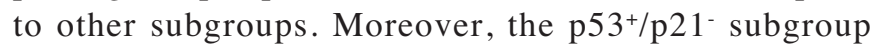
showed a benefit in overall survival after adjuvant chemotherapy as compared to the $\mathrm{p} 53 \% / \mathrm{p} 21^{-}$subgroup. Interestingly, patients with p53-negative tumors were exposed to higher risk $\left(\mathrm{HR}=3.5\right.$ (CI 1.2-9.8) and $\mathrm{HR}_{\mathrm{adj}}=3.6$ (CI 1.2-11.6). It is conceivable that the p53 expression may result in activation of p21 and contribute to cell cycle arrest and apoptosis. Thus, this combined expression status may be the reason for the better outcome in a subgroup of patients with colorectal carcinomas.

Our data suggest that the analysis of p53 expression in combination with other molecular parameters such as p21 might be helpful in assessing patient prognosis in colorectal cancer. The combination of $\mathrm{p} 53^{+} / \mathrm{p} 21^{-}$may also serve as a biomarker for identification of patients who would have a benefit from adjuvant chemotherapy.

\section{Acknowledgements}

We would like to thank Professor Christoph Hanski for helpful discussion and Petra Wachs for technical assistance.

\section{References}

1. Jemal A, Siegel R, Ward E, Murray T, Xu J and Thun MJ: Cancer statistics, 2007. CA Cancer J Clin 57: 43-66, 2007.

2. O'Connell JB, Maggard MA and Ko CY: Colon cancer survival rates with the new American Joint Committee on Cancer sixth edition staging. J Natl Cancer Inst 96: 1420-1425, 2004.

3. Nehls O, Okech T, Hsieh CJ, et al: Studies on p53, BAX and Bcl-2 protein expression and microsatellite instability in stage III (UICC) colon cancer treated by adjuvant chemotherapy: major prognostic impact of proapoptotic BAX. Br J Cancer 96: 1409-1418, 2007.

4. Mamounas E, Wieand S, Wolmark N, Bear HD, Atkins JN, Song $J$ and Rockette H: Comparative efficacy of adjuvant chemotherapy in patients with Dukes' B versus Dukes' C colon cancer: results from four National Surgical Adjuvant Breast and Bowel Project adjuvant studies (C-01, C-02, C-03, and C-04). J Clin Oncol 17: 1349-1355, 1999.

5. Aneja R, Ghaleh AM, Zhou J, Yang VW and Joshi HC: p53 and p21 determine the sensitivity of Noscapine-induced apoptosis in colon cancer cells. Cancer Res 67: 3862-3870, 2007.

6. Gryfe R, Kim H, Hsieh ET, et al: Tumor microsatellite instability and clinical outcome in young patients with colorectal cancer. $\mathrm{N}$ Engl J Med 342: 69-77, 2000.

7. Ribic CM, Sargent DJ, Moore MJ, et al: Tumor microsatelliteinstability status as a predictor of benefit from fluorouracil-based adjuvant chemotherapy for colon cancer. N Engl J Med 349: 247-257, 2003

8. Van Krieken JHJM, Jung A, Kirchner T, et al: KRAS mutation testing for predicting response to anti-EGFR therapy for colorectal carcinoma: proposal for an European quality assurance program. Virchows Archiv 453: 417-431, 2008.

9. Lyall MS, Dundas S, Curran S and Murray GI: Profiling markers of prognosis in colorectal cancer. Clin Cancer Res 12: 1184-1191, 2006.

10. Ricciardiello L, Ceccarelli C, Angiolini G, et al: High thymidylate synthase expression in colorectal cancer with microsatellite instability: implications for chemotherapeutic strategies. Clin Cancer Res 11: 4234-4240, 2005.

11. Hilska M, Collan YU, O Laine VJ, Kössi J, Hirsimäki P, Laato M and Roberts PJ: The significance of tumor markers for proliferation and apoptosis in predicting survival in colorectal cancer. Dis Colon Rectum 48: 2197-2208, 2005.

12. Galizia G, Ferraraccio F, Lieto E, et al: Prognostic value of p27, $\mathrm{p} 53$, and vascular endothelial growth factor in Dukes A and B colon cancer patients undergoing potentially curative surgery. Dis Colon Rectum 47: 1904-1914, 2004.
13. Resnick MB, Routhier J, Konkin T, Sabo E and Pricolo VE: Epidermal growth factor receptor, c-MET, beta-catenin, and p53 expression as prognostic indicators in stage II colon cancer: a tissue microarray study. Clin Cancer Res 10: 3069-3075, 2004.

14. Hall PA and McCluggage WG: Assessing p53 in clinical contexts: unlearned lessons and new perspectives. J Pathol 208: $1-6,2006$.

15. Ogino S, Kawasaki T, Kirkner GJ, Ogawa A, Dorfman I, Loda M and Fuchs CS: Down-regulation of p21 (CDKNIA/CIPI) is inversely associated with microsatellite instability and $\mathrm{CpG}$ island methylator phenotype (CIMP) in colorectal cancer. J Pathol 210: 147-154, 2006.

16. Elsaleh H, Powell B, Soontrapornchai P, Joseph D, Goria F, Spry $\mathrm{N}$ and Iacopetta B: p53 gene mutation, microsatellite instability and adjuvant chemotherapy: impact on survival of 388 patients with Dukes' C colon carcinoma. Oncology 58: 52-59, 2000.

17. Gallego MG, Acenero MJ, Ortega S, Delgado AA and Cantero JL: Prognostic influence of p53 nuclear overexpression in colorectal carcinoma. Dis Colon Rectum 43: 971-975, 2000.

18. Tollenaar R, van Krieken J, van Slooten HJ, et al: Immunohistochemical detection of $\mathrm{p} 53$ and $\mathrm{Bcl}-2$ in colorectal carcinoma: no evidence for prognostic significance. Br J Cancer 77: 1842-1847, 1998.

19. Pereira H, Silva S, Juliao R, Garcia P and Perpetua F: Prognostic markers for colorectal cancer: expression of p53 and bcl2. World J Surg 21: 210-213, 1997.

20. Starzynska T, Bromley M, Marlicz K, Roberts SA, Ucinski M and Stern PL: Accumulation of p53 in relation to long-term prognosis in colorectal carcinoma. Eur J Gastroenterol Hepatol 9: 183-186, 1997.

21. Russo A, Bazan V, Iacopetta B, Kerr D, Soussi T and Gebbia N: The TP53 colorectal cancer international collaborative study on the prognostic and predictive significance of p53 mutation: influence of tumor site, type of mutation, and adjuvant treatment. J Clin Oncol 23: 7518-7528, 2005.

22. Lan YT, Chang SC, Fen-Yau Li A, et al: p53 protein accumulation as a prognostic marker in sporadic colorectal cancer. Int J Colorectal Dis 22: 499-506, 2007.

23. Soong R, Grieu F, Robbins P, et al: p53 alterations are associated with improved prognosis in distal colonic carcinomas. Clin Cancer Res 3: 1405-1411, 1997.

24. Adrover E, Maestro ML, Sanz-Casla MT, del Barco V, Cerdan J, Fernandez C and Balibrea JL: Expression of high p53 levels in colorectal cancer: a favourable prognostic factor. Br J Cancer 81: 122-126, 1999.

25. Bouzourene H, Gervaz P, Cerottini JP, et al: p53 and Ki-ras as prognostic factors for Dukes' stage B colorectal cancer. Eur J Cancer 36: 1008-1015, 2000.

26. Gafa R, Maestri I, Matteuzzi M, Santini A, Farretti S, Cavazzini L and Lanza G: Sporadic colorectal adenocarcinomas with highfrequency microsatellite instability. Cancer 89: 2025-2037, 2000.

27. Schwandner O, Bruch HP and Broll R: p21, p27, cyclin D1, and p53 in rectal cancer: immunohistology with prognostic significance? Int J Colorectal Dis 17: 11-19, 2002.

28. Smyth EF, Sharma A, Sivarajasingham N, Hartley J, Monson JR and Cawkwell L: Prognostic implications of hMLH1 and p53 immunohistochemical status in right-sided colon cancer. Dis Colon Rectum 47: 2086-2091, 2004.

29. Klump B, Nehls O, Okech T, et al: Molecular lesions on colorectal cancer - impact on prognosis? Own data and review of the literature. Int J Colorectal Dis 19: 23-42, 2004.

30. Watanabe T, Wu TT, Catalano PJ, et al: Molecular predictors of survival after adjuvant chemotherapy for colon cancer. N Engl J Med 344: 1196-1206, 2001.

31. Fernebro E, Bendahl PO, Dictor M, Persson A, Fernö M and Nilbert M: Immunohistochemical patterns in rectal cancer: application of tissue microarray with prognostic correlations. Int J Cancer 111: 921-928, 2004.

32. Fu CG, Tominaga O, Nagawa $\mathrm{H}$, et al: Role of $\mathrm{p} 53$ and $\mathrm{p} 21 /$ WAF1 detection in patient selection for preoperative radiotherapy in rectal cancer patients. Dis Colon Rectum 41: 68-74, 1998.

33. Zirbes TK, Baldus SE, Moenig SP, et al: Prognostic impact of p21/waf1/cip1 in colorectal cancer. Int J Cancer (Pred Oncol) 89: 14-18, 2000.

34. Edmonston TB, Cuesta KH, Burkholder S, et al: Colorectal carcinomas with high microsatellite instability: defining a distinct immunologic and molecular entity with respect to prognostic markers. Hum Pathol 31: 1506-1514, 2000. 
35. Prall F, Ostwald C, Nizze H and Barten M: Expression profiling of colorectal carcinomas using tissue microarrays: cell cycle regulatory proteins $\mathrm{p} 21, \mathrm{p} 27$, and p53 as immunohistochemical prognostic markers in univariate and multivariate analysis. Appl Immunohistochem Mol Morphol 12: 111-121, 2004.

36. Mitomi H, Mori A, Kanazawa H, et al: Venous invasion and down-regulation of p21(WAF1/CIP1) are associated with metastasis in colorectal carcinomas. Hepatogastroenterology 52: 1421-1426, 2005.

37. Lebe B, Sarioglu S, Sökmen S, Ellidokuz H, Füzün M and Küpelioglu A: The clinical significance of p53, p21, and p27 expressions in rectal carcinoma. Appl Immunohistochem Mol Morphol 13: 38-44, 2005.

38. Cornfield DB, Palazzo JP, Schwartz GF, et al: The prognostic significance of multiple morphologic features and biologic markers in ductal carcinoma in situ of the breast: a study of a large cohort of patients treated with surgery alone. Cancer 100: 2317-2327, 2004.
39. Ahnen DJ, Feigl P, Quan G, et al: Ki-ras mutation p53 overexpression predict the clinical behavior of colorectal cancer: A Southwest Oncology Group Study 1. Cancer Res 58: 1149$1158,1998$.

40. Munro AJ, Lain S and Lane DP: P53 abnormalities and outcomes in colorectal cancer: a systematic review. Br J Cancer 92: 434-444, 2005.

41. Schelwies K, Sturm I, Grabowski P, et al: Analysis of p53/BAX in primary colorectal carcinomas: low BAX protein expression is a negative prognostic factor in UICC stage III tumours. Int J Cancer 99: 589-596, 2002.

42. Rau B, Sturm I, Lage H, et al: Dynamic expression profile of p21WAF1/CIP1 and Ki-67 predicts survival in rectal carcinoma treated with preoperative radiochemotherapy. J Clin Oncol 21: 3391-3401, 2003.

43. Mitomi H, Ohkura Y, Fukui N, et al: p21 ${ }^{\mathrm{WAF} / \mathrm{CIP} 1}$ expression in colorectal carcinomas is related to Kras mutations and prognosis. Eur J Gastrol Hepatol 19: 883-889, 2007. 